\title{
Mobile Ad Hoc Networks: the DHT paradigm
}

\author{
Marcello Caleffi \\ Dipartimento di Ingegneria Biomedica, Elettronica e delle Telecomunicazioni (DIBET) \\ Università degli Studi di Napoli Federico II - Napoli, ITALY \\ marcello.caleffi@unina.it
}

\begin{abstract}
In this paper, a DHT-based routing protocol which integrates at the network layer both traditional direct routing, i.e. MANET routing, and indirect key-based routing, i.e. P2P routing, has been proposed. The unique feature of such a proposal is the ability to build an overlay network in which both the logical and physical proximity agree, improving so the $P 2 P$ performances. In addition, it outperforms traditional routing protocols for MANET communications whenever the number of nodes grows, assuring satisfactory performances also for large networks operating in presence of hostile channels and moderate node mobility.
\end{abstract}

\section{OVERVIEW}

Mobile Ad hoc NETworks (MANETs) and Peer-To-Peer (P2P) systems are emerging technologies sharing a common underlying decentralized networking paradigm. Despite the similarities, the adoption of the $\mathrm{P} 2 \mathrm{P}$ paradigm to disseminate and discover information in a MANET scenario rises to new and challenging problems due to the layer where they operate: P2Ps build and maintain overlay networks at the applicationlayer, assuming the presence of an underlying network routing which assures connectivity among nodes, while MANETs focus on providing a multi-hop wireless connectivity among nodes. It has been proved that simply deploying P2P over MANETs may cause poor performances due to the lack of cooperation and communication between the two layers. For these reasons, different cross-layer approaches have been presented [1]-[4]. Most of them associate an identifier, namely a key, to each peer by means of an hash function and organize the keys in a certain structure, for example a ring. Since the identifiers are randomly assigned to peers, the P2P overlay topology is usually built independently from the physical one, and thus no relationship exists between overlay and physical proximities (Fig. 1), producing so unnecessary long routes.

In the paper, we present a DHT-based routing protocol, namely Indirect Tree-based Routing (ITR), which integrates both traditional direct routing and indirect key-based routing at the network layer and provide a fully functional P2P network [5]. ITR assigns to peers location-dependent identifiers, namely strings of $l$ bits, by means of a distribute procedure which assures that nodes, which share the same id prefix, forms a connected sub-graph in the network topology [6].

This work is partially supported by the Italian National Project "Wireless multiplatfOrm mimo active access netwoRks for QoS-demanding muLtimedia Delivery" (WORLD) under grant number 2007R989S, and by the Regional Project "REmote e COntinuous Monitoring" (RECOM).

The author would like to thanks the advisor, Prof. Luigi Paura, for his trust and unwavering support.

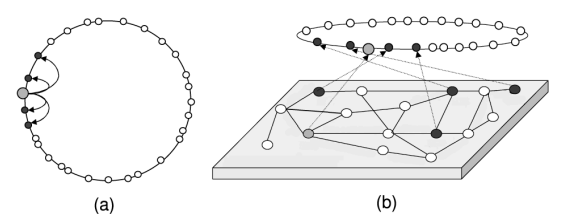

Fig. 1. Traditional P2P overlay network

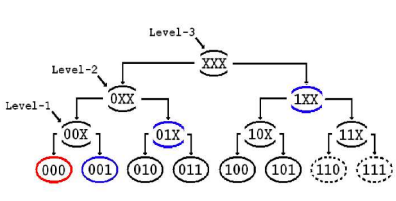

(a)

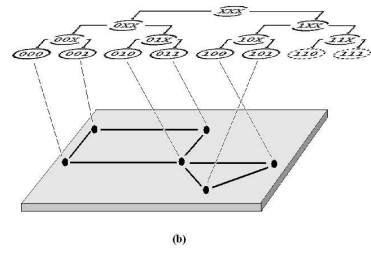

Fig. 2. ITR overlay network
By means of such a property, the MANET routing is notably simplified: each routing table consists of $l$ sections (one for each bit of the id) and forwarding a packet consists of the selection of the best entry (according to a certain metric) stored in the $k$ section, where $k$ is the address prefix length shared by the forwarder and the destination ids. Moreover, the P2P routing benefits from the location-dependent ids as well, since they are assigned taking into account the network topology, and thus the logical proximity agree with the physical one, as shown in Fig. 2. In other words, ITR is able to forward resource queries without introducing any overlay overhead.

\section{Performance Analysis}

To test the effectiveness of our proposal, we have adopted the widely adopted network simulator ns- 2 and, for sake of brevity, only a subset of the experiments [5] is here reported. The first scenario models a realistic 802.11 b ad hoc network in order to assess the performances of ITR in terms of traditional routing, by comparing them with those of three well-known MANET protocols, namely AODV, DSR and DSDV [7]. The channel model accounts for long-term fading effects, additive thermal noise and mutual interference, while the data pattern is random: each node select a destination for a CBR over UDP flow with 1000 byte as packet size according to a uniform distribution. To effectively assess the scalability property of the analyzed protocols [8], the data throughput generated by each source is defined as $\lambda=\frac{W}{n \sqrt{n}}$, where $W$ is the link data throughput and $n$ is the number of nodes. The results 


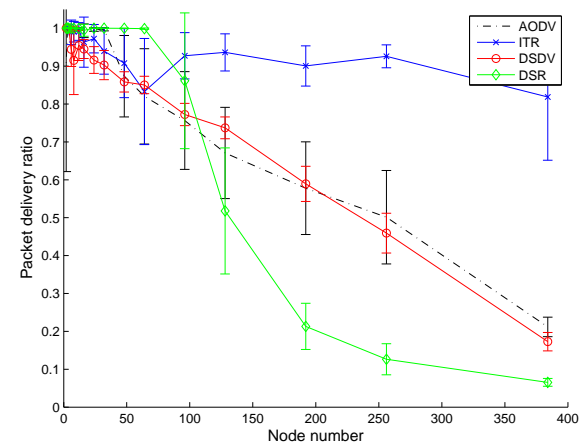

Fig. 3. Packet Delivery Ratio

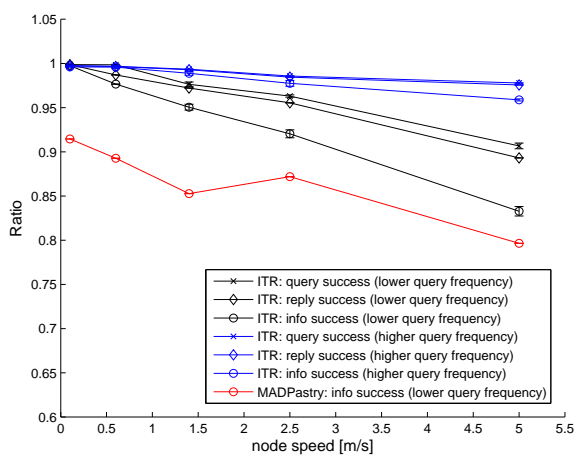

Fig. 4. Success rates

for such a scenario are illustrated by Fig. 3, which shows that ATR performances remains largely unaffected by the node number. On the other hand, DSDV and AODV performances decrease rough linearly with the number of nodes. Finally, as the nodes number increases, DSR performances become the worst and, with reference to largest networks, nearly an order of magnitude separates them from ATR ones.

The second scenario models an ideal 802.11b MANET in order to assess the performances of ITR in terms P2P routing, by comparing them with those obtained by a widely adopted protocol, namely MADPastry [4]. The channel model are the two-ray ground one and 50 nodes move in accordance with the random way-point model with no pause time and at a steady speed. The P2P traffic pattern is random: each node stores 100 resources and it sends periodically a query for a resource selected according to a uniform distribution. To explore the impact of the caching techniques (each resource pointer is cached for 10 seconds), we set the resource-query frequency for ITR to both 0.1 and 0.5 query/s, while the query-frequency for MADPastry is set to 0.1. The adopted metrics in the second scenario are the i) query success rate, i.e. the fraction of resource queries correctly delivered to the rendezvouspoint, ii) reply success rate, i.e. the ratio between the number of resource replies correctly delivered and the number of generated resource queries, iii) resource success rate, i.e. the ratio between the number of resources correctly delivered

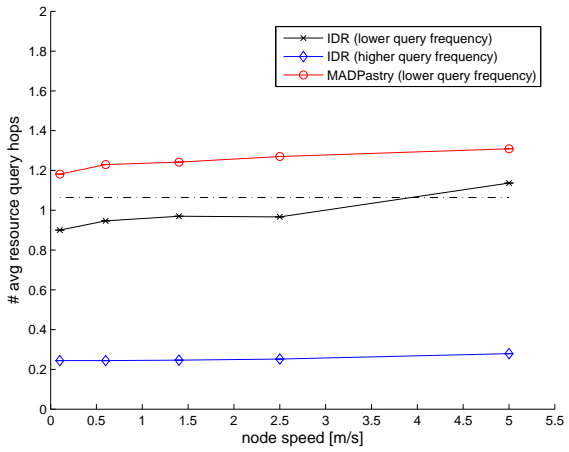

Fig. 5. Path length

and the number of generated resource queries. Moreover, we evaluate also the average hop number of resource queries to assess the ability of a P2P protocol to effectively build a physical proximity-aware overlay network. The results in terms of success ratios are shown in Fig. 4: ITR outperforms MADPastry for the considered speed values, and, in presence of cache hits, ITR is able to delivery all the queries to the correct rendezvous-point as well as to retrieve all the required resources. Fig. 5 shows the results in terms of resource query hop count. As regards to ITR, in absence of caching techniques the average overlay hop number does not significant differ with the average physical hop number (equals to 1.06), while MADPastry exhibits an overlay stretch effect.

\section{CONCLUSION}

This paper proposes a scalable DHT-based routing protocol able to effectively build a P2P system in which the logical proximity agrees with the physical proximity, by resorting to a cross-layer approach between network and application layers. The motivation behind this work was to challenge the status quo in mobile peer to peer research, and currently we are working to extend ITR to work in scenarios characterized by high mobility, resorting to the opportunistic routing paradigm.

\section{REFERENCES}

[1] H. Pucha, S. M. Das, and Y. C. Hu, "Ekta: an efficient dht substrate for distributed applications in mobile ad hoc networks," in WMCSA 2004, 2004, pp. 163-173.

[2] A. Rowstron and P. Druschel, "Pastry: Scalable, decentralized object location and routing for large-scale peer-to-peer systems," in IFIP/ACM International Conference, Nov. 2001, pp. 329-350.

[3] M. Caesar, M. Castro, E. Nightingale, G. O'Shea, and A. Rowstron, "Virtual ring routing: network routing inspired by dhts," in SIGCOMM '06, 2006, pp. 351-362.

[4] T. Zahn and J. Schiller, "MADPastry: A DHT Substrate for Practicably Sized MANETs," in ASWN2005, June 2005.

[5] M. Caleffi, "Mobile ad hoc networks: the dht paradigm, ph.d. thesis," November 2008.

[6] M. Caleffi, G. Ferraiuolo, and L. Paura, "Augmented tree-based routing protocol for scalable ad hoc networks," in MASS 2007, Oct. 2007, pp. $1-6$.

[7] J. Broch, D. Maltz, D. Johnson, Y. Hu, and J. Jetcheva, "A performance comparison of multi-hop wireless ad hoc network routing protocols," in MobiCom '98, 1998, pp. 85-97.

[8] P. Gupta and P. Kumar, "The capacity of wireless networks," Information Theory, IEEE Transactions on, vol. 46, no. 2, pp. 388-404, Mar 2000. 